\title{
Association of temporomandibular disorder-related pain with severe headaches-a Bayesian view
}

\author{
Javed Ashraf ${ }^{1}$ (i) $\cdot$ Matti Närhi ${ }^{1,2} \cdot$ Anna Liisa Suominen ${ }^{1,3,4} \cdot$ Tuomas Saxlin $^{1,3}$
}

Received: 30 April 2021 / Accepted: 22 June 2021 / Published online: 5 July 2021

(c) The Author(s) 2021

\begin{abstract}
Objectives Association of temporomandibular disorders (TMD)-related pain with severe headaches (migraine and tensiontype headaches $[\mathrm{TTH}]$ ) was studied over a follow-up period of 11 years.

Materials and methods The data used was from two nationally representative health surveys in Finland-the Health 2000 Survey (baseline) and the Health 2011 Survey (follow-up) (Bioresource Research Impact Factor [BRIF] 8901)—conducted by the Finnish Institute for Health and Welfare (THL). The primary dataset of the current study included a subset of the population undergoing a clinical oral examination, including TMD examination, at baseline, and answering the questions related to severe headaches, both at baseline and at follow-up $(n=530)$. From the primary dataset, two datasets were created to study the onset of migraine (dataset 1) and TTH (dataset 2) separately. Dataset 1 included participants healthy of migraine, but not other headaches, at baseline $(n=345)$, and dataset 2 participants healthy of TTH and other headaches, except migraine, at baseline $(n=464)$. Bayesian logistic regression models with weakly informative priors were utilized to assess the association of muscle-related TMD pain (mTMD) at baseline and temporomandibular joint-related TMD pain (jTMD) at baseline with the presence of migraine and TTH at follow-up.

Results Neither of the baseline TMD-related pain variables were associated with the presence of migraine at follow-up (posterior effect estimates-0.12, 95\% credible interval [CI] -0.49-0.24, and 0.11, 95\% CI-0.38-0.59, for mTMD and jTMD, respectively), whereas $\mathrm{mTMD}$ at baseline (posterior effect estimate $0.36,95 \% \mathrm{CI} 0.02-0.69$ ), but not jTMD at baseline (posterior effect estimate -0.32, 95\% CI-0.94-0.25), was associated with the presence of TTH at follow-up. Bayesian sensitivity analyses revealed that the estimates of the regression models were stable, demonstrating sufficient validity and consistency of the estimates.

Conclusion These results indicate that diverse mechanisms may exist behind the associations of TMD-related painful conditions with different types of severe headaches.

Clinical relevance TMD-related pain is a frequent comorbidity of severe primary headaches. Therapy of severe primary headaches may thus benefit significantly with the incorporation of a multi-disciplinary clinical team.
\end{abstract}

Keywords Temporomandibular disorders · Headache disorders · Pain · Bayesian logistic regression · Sensitivity analysis · Directed acyclic graphs

Javed Ashraf

javed.ashraf@uef.fi

1 Institute of Dentistry, University of Eastern Finland, P.O. Box 1627, 70211 Kuopio, Finland

2 Institute of Biomedicine, University of Eastern Finland, Kuopio, Finland

3 Department of Oral and Maxillofacial Diseases, Kuopio University Hospital, Kuopio, Finland

4 Department of Public Health and Welfare, the Finnish Institute for Health and Welfare, Helsinki, Finland

\section{Introduction}

The prevalence of temporomandibular disorder (TMD) symptoms varies significantly between populations. A recent systematic review indicated that in general populations the prevalence of having at least one clinical sign of TMD ranges between 5 and $60 \%$ [1]. Nonetheless, pain in the temporomandibular region is a common clinical sign, occurring in approximately $10 \%$ of the adult population [2]. In Finland, a recent study reported that at least one-third $(34.6 \%)$ of the Finnish population suffered from at least 
one clinical sign of TMD. This study also reported that the prevalence of muscle-related TMD pain (mTMD) was $1.9 \%$ and $6.5 \%$ for males and females, and the prevalence of temporomandibular joint-related TMD pain (jTMD) was $1.7 \%$ and 3.5\% for males and females, respectively [3].

Primary headaches (migraine and tension-type headaches [TTH]), on the other hand, affect more than 2.5 billion individuals worldwide. A recent global study ranked headaches as the second leading cause of years lost due to disability after lower back pain [4]. Globally, the number of individuals suffering from migraine and TTH in the year 2017 was estimated to be 1.3 and 2.3 billion, and the percentage of increase of those suffering from these disorders within the decade 2007-2017 was $15 \%$ and $16 \%$, respectively [5]. In Finnish population-according to a recent retrospective study based on the electronic medical records of Finland's largest private occupational health care provider-the overall prevalence of migraine was $7 \%$ in females and $2 \%$ in males [6]. Moreover, the proportion of TTH sufferers in Finland has been amounted to $16 \%$ of the total population [7].

TMD-related pain (mTMD and jTMD) seldom occurs in isolation; approximately only $17 \%$ of the TMD-related pain cases occur with no other comorbid pain condition [8]. TMD-related pain is often associated with other chronic pains causing significant physical and psychological disability [9]. Amongst the comorbidities of the TMD-related pain, headaches - such as migraine and TTH—are frequently reported along with both mTMD and jTMD [10]. Association of mTMD with migraine has been reported also in a recent cross-sectional study based on a representative study sample of adult Finnish population [11].

To the best of the authors' knowledge, all the previous studies of the association of TMD-related pain with headaches have been based on the Frequentist statistics. Compared to the Bayesian approach, the Frequentist statistics suffer from some limitations, most importantly the dependence on large sample sizes for effect sizes to be accurately determined [12]. Additionally, in contrast to the Frequentist methodology, the Bayesian statistics do not provide one (fixed) outcome value but rather an interval containing the regression coefficient [13]. These intervals, termed credible intervals (CI), attribute a probability to the best estimate and to all the possible values of the parameter estimates [12].

The current study-utilizing the Bayesian methodology-aimed to examine the association of TMD-related pain with severe headaches (migraine and TTH) over a follow-up period of 11 years. The hypothesis was that a prospective association exists between TMD-related pain and severe headaches.

\section{Materials and methods}

\section{Study sample and participants}

The current study utilized data from the Health 2000 (baseline) and the Health 2011 (follow-up) Surveys (Bioresource Research Impact Factor [BRIF] 8901), conducted by the Finnish Institute for Health and Welfare (THL) (former National Public Health Institute [KTL] of Finland).

The Health 2000 Survey, conducted in the years 2000 and 2001, included 9922 invited participants aged 18 years or older living in mainland Finland. The data for this survey were collected through self-administered questionnaires and interviews, and for those aged 30 years or older also by clinical oral and health examinations as well as by laboratory analyses. The participation rate of the Health 2000 Survey was $92 \%(n=9125)$ (participation in at least one phase of the survey) [14].

The Health 2011 Survey was a follow-up study of the Health 2000 Survey, fieldwork of which was conducted in the years 2011-2012. The invited participants of the Health 2011 Survey included those participants of the Health 2000 Survey sample who were alive, living in Finland, had contact details available, and not refused to participate in further surveys previously $(n=8135)$, participation rate in at least one phase of the survey being $73 \%(n=5903)$. The main reason for non-participation was the refusal to participate, following inability to contact the participant, the death of the invited participant, and the invited participant living abroad. The Health 2011 Survey included many smaller studies specific to a common disorder/group of disorders. Participants reporting to ever suffer from severe (moderate to intense) headaches during the health interview of the Health 2011 Survey proper $(n=875)$ were invited to participate one of the smaller studies, namely Migraine Sub-study. It included questions on headache types, severity, and their impact. Of the eligible participants, 832 eventually participated (95\%) [15].

The current study utilized a subset of the population undergoing clinical oral examination, including TMD examination, at baseline (the Health 2000 Survey) and answering the questions related to severe headaches both at baseline and at follow-up (the Migraine Sub-study of the Health 2011 Survey) $(n=530)$. From this subset, two datasets were created to study the onset of migraine (dataset 1) and TTH (dataset 2), and were analyzed separately. Dataset 1 included only the participants healthy of migraine, but not other headaches, at baseline (reported in the interview of not having migraine diagnosed by a physician in the Health 2000 Survey) $(n=345)$, and dataset 2 only the participants not suffering from TTH or other headaches, except migraine, at baseline (reported in the 
interview of not having TTH or other headaches [migraine excluded] diagnosed by a physician in the Health 2000 Survey) ( $n=464)$ (Fig. 1).

\section{Outcome variables}

The outcome variables were the presence (yes/no) of migraine or TTH at follow-up (the Health 2011 Survey). These were asked by questions: "Did a physician ever diagnose you for migraine?" and "Did a physician ever diagnose you for tension-type headaches?" [15].

\section{Predictor variables}

In the Health 2000 Survey, five experienced and calibrated dentists performed a standardized clinical oral examination. This included TMD examination partly based on the Research Diagnostic Criteria for TMD (RDC-TMD) Axis I criteria [16], focusing on the most central clinical aspects. The TMD examination involved the palpation of the masticatory muscles ( $m$. temporalis and $m$. masseter) and the temporomandibular joint (TMJ). The pain response during the inspection of the temporomandibular area was recorded dichotomously (yes/no). Further details regarding the TMD examination methodology and protocol can be found at Suominen-Taipale et al. (2008). TMD-related pain variables, mTMD (yes/no) and jTMD (yes/no), were used as the predictor variables. The percentage of agreement for recording pain responses during the TMD examination between the examiners and the reference examiner was 95\% (kappa value 0.47 ; 95\% CI 0.41-0.53) for mTMD and 92\% (kappa value 0.26 ; $95 \%$ CI 0.19-0.34) for jTMD [17].

\section{Other covariates}

Other covariates and potential confounders of the current study were utilized from the baseline data (the Health 2000 Survey). These included gender and age taken from the population registers. Variables recorded during the home-visit interview included the level of education (education below upper secondary or vocational school level [low]; graduated from upper secondary school or vocational school [medium]; and graduated from a university or a polytechnic institute [high]), and the use of anti-inflammatory drugs (yes/no). Body mass index (BMI) was calculated using the weight and height of the study participants assessed during the health
Fig. 1 Schematic representation of the sample utilized in the study

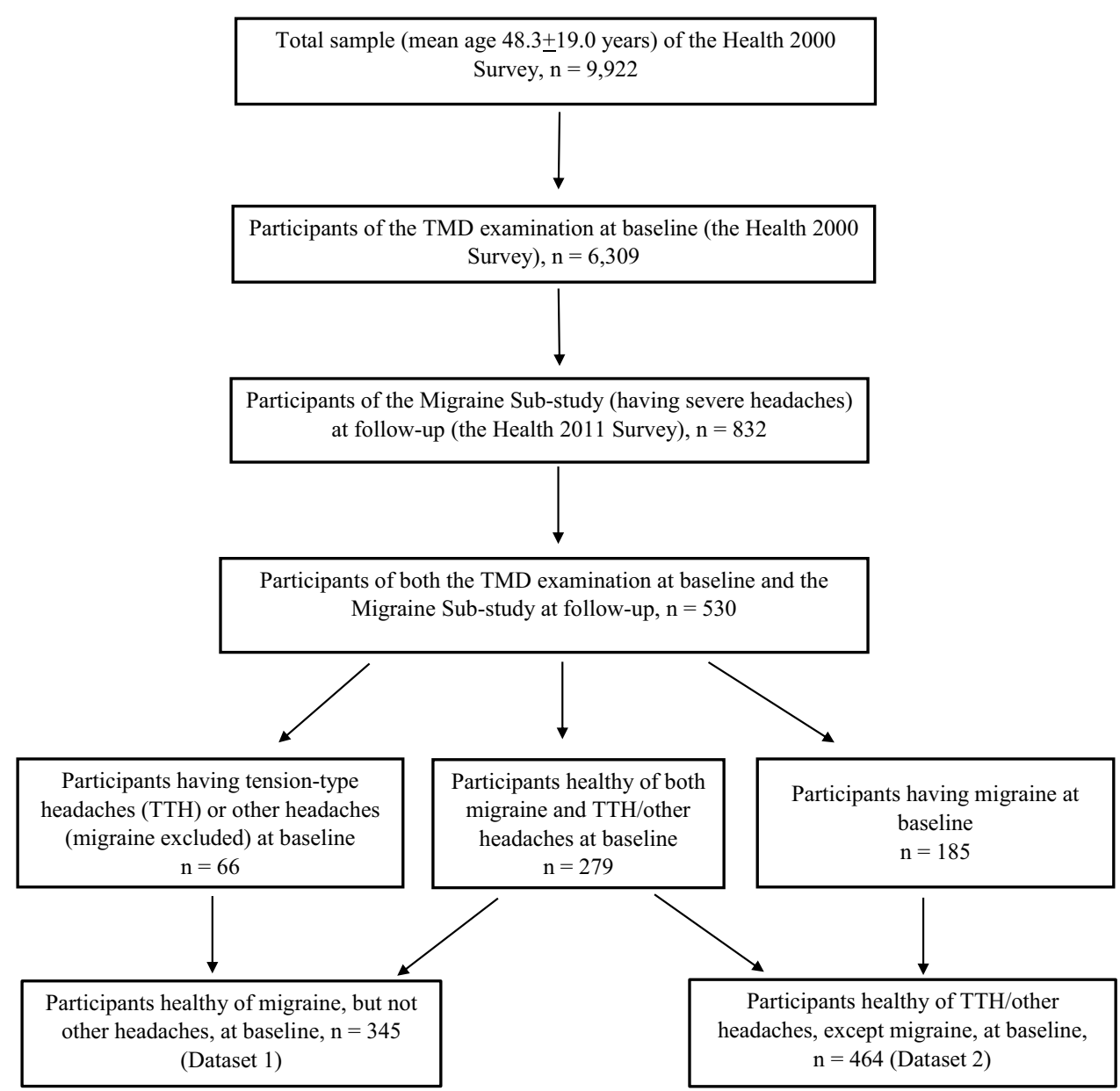


examination (if not available, information from a questionnaire was used). BMI was used as a continuous variable in the analyses [18].

\section{Statistical analyses}

Differences in the frequencies of the categories of the predictor variables and the categorized covariates between those participants reporting $v s$. those not reporting migraine at follow-up, as well as between the TTH and non-TTH study participants at follow-up, were analyzed using chi-square test. Regarding the continuous covariates, the normality of the distributions in different categories of the outcome variables was assessed visually through distribution histograms and tested with Kolmogorov-Smirnov and Shapiro-Wilk tests. Based on these, the Mann-Whitney U test was used to observe differences amongst them in the different outcome variable categories. The $\mathrm{p}$-values for the descriptive statistics of the study population were based on two-tailed tests of significance.

The current study employed directed acyclic graphs (DAG) for the evaluation of the study hypothesis, based on a priori knowledge (Fig. 2a and b). Associations of the potential confounders and covariates with either or both the predictor and the outcome variables in the DAG models were adjusted for in the regression models. These adjustments were made through blocking all the backdoor paths and implementing the disjunctive cause criterion in the DAG [19].

Multivariate Bayesian logistic regression analyses were performed for analyzing the effect estimates between the predictor and the outcome variables. Weakly informative priors (a normal distribution with the mean of zero and the variance of five) were utilized for the regression models in both datasets due to the lack of substantial longitudinal evidence regarding the association of TMD-related pain with severe headaches. Another reason was the better computational stability of the Bayesian algorithm incorporating weakly informed priors in the case of logistic regression models [20]. The effect sizes of the association of TMDrelated pain with severe headaches were estimated using predictive (regression) models. All the analyses of the current study reported the posterior effect estimates (regression coefficients; mean parameter value $\mu$ ) with their Bayesian 95\% credible intervals (CI). Bayesian sensitivity analyses were performed with varying prior distributions for the predictor variables to check for the reliability and stability of the parameter estimate distributions. Visual inspection of the estimation convergence of all regression models was done through graphical summaries of Bayesian parameter trace plots, Bayesian posterior parameter distribution plots, and Bayesian autocorrelation plots. A posterior predictive p-value of $>0.05$ was taken as a good model fit for all the

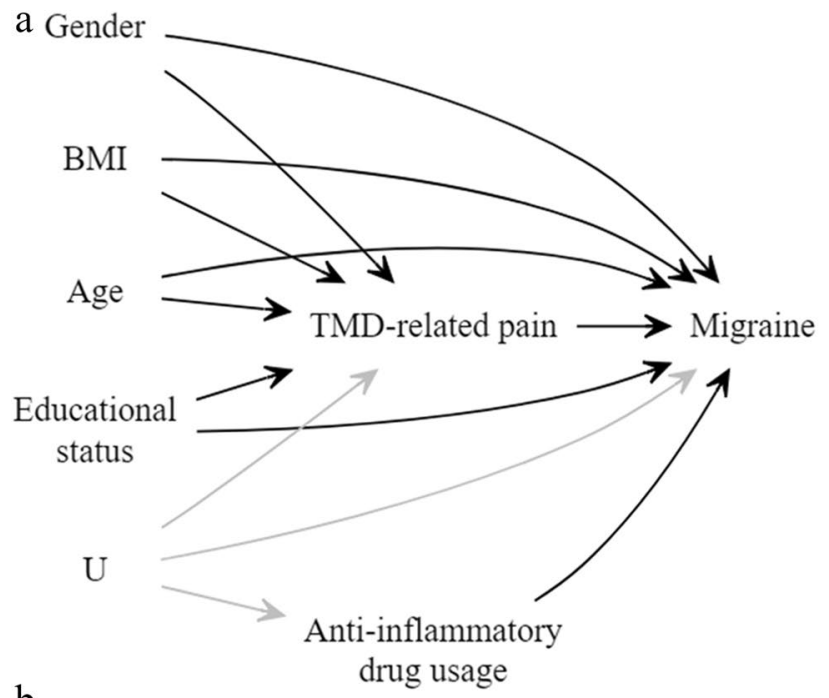

b
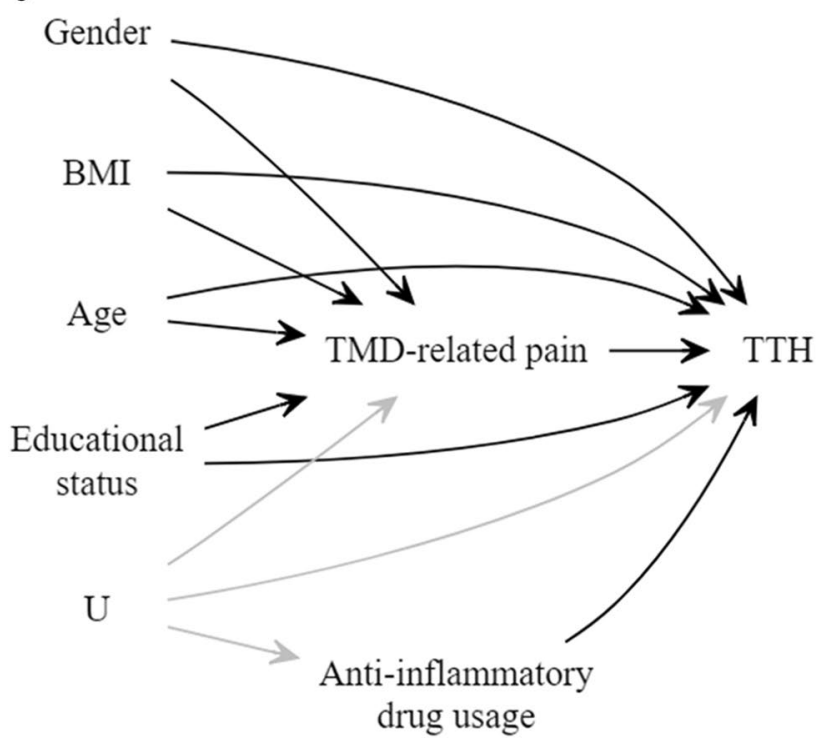

Fig. 2 a Directed acyclic graphs depicting the hypothetical model for the associations between TMD-related pain, covariates, and the presence of migraine. $\mathrm{U}$, unmeasured confounder, i.e., psychological status. Grey arrows = denoting disjunctive cause between the outcome variable and the covariate of anti-inflammatory drug usage through unmeasured confounder of psychological status. b Directed acyclic graphs depicting the hypothetical model for the associations between TMD-related pain, covariates, and the presence of tension-type headaches (TTH). U, unmeasured confounder, i.e., psychological status. Grey arrows $=$ denoting disjunctive cause between the outcome variable and the covariate anti-inflammatory drug usage through unmeasured confounder of psychological status

regression models. Complete case analyses was adhered to in the regression analyses; the missing data was handled by excluding all the study participants with incomplete data from the analyses.

SPSS (IBM Corp., Armonk, NY, USA) version 27 was used for the descriptive data analyses. Bayesian regression and sensitivity analyses were performed through 
Mplus version 8.4 (Muthen \& Muthen, 1998-2019, Los Angeles, CA, USA). DAGs and flow charts for the current study were drawn through DigrammeR package of $R$ software (http://www.R-project.org/).

\section{Ethical issues}

Both surveys were based on voluntariness, and all the participants gave their written informed consent before their participation. Ethical approval for the Health 2000 Survey was obtained from the Ethics Committee for Epidemiology and Public Health of the Hospital District of Helsinki and Uusimaa, Finland. Ethical approval for the Health 2011 Survey was obtained from the Coordinating Ethics Committee at the Hospital District of Helsinki and Uusimaa, Finland [15, 18].

Guideline from the STROBE Statement—checklist was adhered to in the current manuscript.

\section{Results}

Basic characteristics of the participants with $(n=269)$ and without $(n=261)$ migraine at follow-up, and participants with $(n=105)$ and without TTH $(n=425)$ at follow-up are presented in Tables 1 and 2, respectively.

In the regression analyses of the dataset 1 -after adjusting for potential confounding factors such as gender, age, the level of education, BMI, and the use of anti-inflammatory drugs-the $95 \%$ CI of the posterior effect estimates of both mTMD and jTMD at baseline in the association with the presence of migraine at follow-up included null values, thus denoting no association between these variables (Table 3).

According to the regression analyses of the dataset 2, after adjusting for confounding factors, mTMD at baseline was found to predict the presence of TTH at follow-up (posterior effect estimate $0.36,95 \%$ CI $0.02-0.69$ ). However, null value was included in the $95 \% \mathrm{CI}$ of the posterior effect estimate of jTMD at baseline in the association with the presence of TTH at follow-up suggesting no association (Table 3).
Table 1 Characteristics of the study participants at baseline (the Health 2000 Survey) by the presence of migraine at follow-up (Health 2011 Survey), dataset $1, \mathrm{n}=345$

\begin{tabular}{|c|c|c|c|c|}
\hline & \multicolumn{4}{|c|}{ Presence of migraine at follow-up } \\
\hline & Yes & No & Total, n (\%) & $p$-value \\
\hline Muscle-related TMD pain, n (\%) & & & & $1.00^{*}$ \\
\hline Yes & $22(19.5)$ & $46(19.8)$ & $68(19.7)$ & \\
\hline No & $91(80.5)$ & $186(80.1)$ & $277(80.3)$ & \\
\hline Total & $113(100)$ & $232(100)$ & $345(100)$ & \\
\hline Joint-related TMD pain, $\mathrm{n}(\%)$ & & & & $0.55^{*}$ \\
\hline Yes & $12(10.6)$ & $19(8.2)$ & $31(9.0)$ & \\
\hline No & $101(80.4)$ & $213(91.8)$ & $314(91.0)$ & \\
\hline Total & $113(100)$ & $232(100)$ & $345(100)$ & \\
\hline Gender, n (\%) & & & & $<0.01^{*}$ \\
\hline Males & $20(17.7)$ & $92(39.7)$ & $112(32.5)$ & \\
\hline Females & $93(82.3)$ & $140(60.3)$ & $233(67.5)$ & \\
\hline Total & $113(100)$ & $232(100)$ & $345(100)$ & \\
\hline Age, median (SD) & $44.4(10.6)$ & $46.6(11.7)$ & & $0.14^{\dagger}$ \\
\hline Education level, n (\%) & & & & $0.39 *$ \\
\hline Low & $28(24.8)$ & $53(22.8)$ & $81(23.5)$ & \\
\hline Medium & $34(30.1)$ & $87(37.5)$ & $121(35.1)$ & \\
\hline High & $51(45.1)$ & $92(39.7)$ & $143(41.4)$ & \\
\hline Total & $113(100)$ & $232(100)$ & $345(100)$ & \\
\hline BMI, mean (SD) & $26.0(4.5)$ & $26.1(4.0)$ & & $0.89^{\dagger}$ \\
\hline Use of anti-inflammatory drugs, n (\%) & & & & $0.03 *$ \\
\hline Yes & $63(58.9)$ & $102(46.4)$ & $165(50.5)$ & \\
\hline No & $44(41.1)$ & $118(53.6)$ & $162(49.5)$ & \\
\hline Total & $107(100)$ & $220(100)$ & $327(100)$ & \\
\hline
\end{tabular}


Table 2 Characteristics of the study participants at baseline (the Health 2000 Survey) by the presence of tension-type headaches (TTH) at follow-up (Health 2011 Survey), dataset $2, \mathrm{n}=464$

\begin{tabular}{|c|c|c|c|c|}
\hline & \multicolumn{4}{|c|}{ Presence of TTH at follow-up } \\
\hline & Yes & No & Total, n (\%) & $p$-value \\
\hline Muscle-related TMD pain, n (\%) & & & & $0.05 *$ \\
\hline Yes & $24(27.3)$ & $66(17.6)$ & $90(19.4)$ & \\
\hline No & $64(72.7)$ & $310(82.4)$ & $374(80.6)$ & \\
\hline Total & $88(100)$ & $376(100)$ & $464(100)$ & \\
\hline Joint-related TMD pain, n (\%) & & & & $0.48 *$ \\
\hline Yes & $4(4.5)$ & $28(7.4)$ & $32(6.9)$ & \\
\hline No & $84(95.5)$ & $348(92.6)$ & $432(93.1)$ & \\
\hline Total & $88(100)$ & $376(100)$ & $464(100)$ & \\
\hline Gender, n (\%) & & & & $0.01 *$ \\
\hline Males & $14(15.9)$ & $111(29.5)$ & $125(26.9)$ & \\
\hline Females & $74(84.1)$ & $265(70.5)$ & $339(73.1)$ & \\
\hline Total & $88(100)$ & $376(100)$ & $464(100)$ & \\
\hline Age, mean (SD) & $44.3(9.2)$ & $46.0(10.8)$ & & $0.31^{\dagger}$ \\
\hline Education level, n (\%) & & & & $<0.01^{*}$ \\
\hline Low & $9(10.2)$ & $92(24.5)$ & $101(21.8)$ & \\
\hline Medium & $23(26.1)$ & $132(35.1)$ & $155(33.4)$ & \\
\hline High & $56(63.6)$ & $152(40.4)$ & $208(44.8)$ & \\
\hline Total & $88(100)$ & $376(100)$ & $464(100)$ & \\
\hline BMI, mean (SD) & $25.5(4.8)$ & $26.1(4.1)$ & & $0.08^{\dagger}$ \\
\hline Use of anti-inflammatory drugs, n (\%) & & & & $0.47 *$ \\
\hline Yes & $51(60.0)$ & $200(54.9)$ & $251(55.9)$ & \\
\hline No & $34(40.0)$ & $164(45.1)$ & $198(44.1)$ & \\
\hline Total & 85 (100) & $364(100)$ & 449 (100) & \\
\hline
\end{tabular}

${ }^{*} p$-value calculated through chi-square test

${ }^{\dagger} p$-value calculated through Mann-Whitney U test
Table 3 Bayesian logistic regression analyses of the association of TMD-related pain (mTMD and jTMD) with the presence of migraine and tension-type headaches (TTH) at follow-up

\begin{tabular}{clll}
\hline Predictor & $\begin{array}{l}\text { Posterior effect } \\
\text { estimate }\left(\mathrm{SD}^{\dagger}\right)\end{array}$ & $95 \%$ credible interval & $\begin{array}{l}\text { Posterior } \\
\text { predictive } \\
p \text {-value }\end{array}$ \\
\hline $\begin{array}{l}\left.\text { Dataset } 1^{\ddagger} \text { (effective } \mathrm{n}=327\right) \\
\text { mTMD }\end{array}$ & $-0.12(0.19)$ & $-0.49-0.24$ & 0.20 \\
jTMD & $0.11(0.25)$ & $-0.38-0.59$ & 0.34 \\
Dataset $2^{\S}($ effective $\mathrm{n}=449)$ & & 0.13 \\
mTMD & $0.36(0.17)$ & $0.02-0.69$ & 0.16 \\
jTMD & $-0.32(0.30)$ & $-0.94-0.25$ & \\
\hline
\end{tabular}

Adjusted for gender, age, the level of education, body mass index (continuous), and the use of anti-inflammatory drugs

${ }^{\dagger}$ Standard deviation

${ }^{\ddagger}$ Migraine at follow-up as the dependent variable

${ }^{\S} \mathrm{TTH}$ at follow-up as the dependent variable

All the regression models displayed a posterior predictive p-value $>0.05$, suggesting an acceptable fit of the models (Table 3). Additionally, all the regression models demonstrated good convergence, low autocorrelation, and near-to-normal posterior distributions upon visual inspections of the Bayesian posterior parameter trace plots, Bayesian autocorrelation plots, and Bayesian posterior parameter distributions, respectively. According to the Bayesian sensitivity analyses, the effect estimates of all the regression models were stable, demonstrating sufficient validity and reliability of the effect estimates. The sensitivity analyses depicted less than $1 \%$ change in the posterior effect estimates upon the introduction of different prior ranges for the predictor variables (Table 4).

\section{Discussion}

The current study reported that mTMD at baseline predicts the presence of TTH at follow-up. However, no consistent association was found between jTMD at baseline and the presence of TTH at follow-up. In addition, neither of the TMD-related pain variables at baseline were found to be associated with the presence of migraine at follow-up.

A key strength of the current study is the measurement of the predictor variables mTMD and jTMD through clinical TMD examination with good inter-examiner reliability. 
Table 4 Sensitivity analyses of the association of TMD-related pain (mTMD and jTMD) with the presence of migraine and tension-type headaches (TTH) at follow-up

\begin{tabular}{|c|c|c|c|c|}
\hline Predictor & $\begin{array}{l}\text { Default priors }{ }^{\dagger}, \mathrm{N} \\
\left(0,10^{10}\right)\end{array}$ & $\begin{array}{l}\text { Current study priors, } \\
\mathrm{N}(0,5)^{\ddagger}\end{array}$ & Testing priors, $\mathrm{N}(0,10)$ & Testing priors, $\mathrm{N}(2,10)$ \\
\hline \multicolumn{5}{|l|}{ Dataset $1^{\S}$ (effective $\mathrm{n}=327$ ) } \\
\hline \multicolumn{5}{|l|}{ mTMD } \\
\hline Posterior effect estimate (SD) & $-0.12(0.19)$ & $-0.12(0.19)$ & $-0.12(0.19)$ & $-0.12(0.19)$ \\
\hline \multicolumn{5}{|l|}{ jTMD } \\
\hline Posterior effect estimate (SD) & $0.11(0.25)$ & $0.11(0.25)$ & $0.11(0.25)$ & $0.12(0.25)$ \\
\hline \multicolumn{5}{|l|}{ Dataset $2^{\mathbb{I}}($ effective $\mathrm{n}=449)$} \\
\hline \multicolumn{5}{|l|}{ mTMD } \\
\hline Posterior effect estimate (SD) & $0.36(0.17)$ & $0.36(0.17)$ & $0.36(0.17)$ & $0.37(0.17)$ \\
\hline \multicolumn{5}{|l|}{ jTMD } \\
\hline Posterior effect estimate (SD) & $-0.32(0.30)$ & $-0.32(0.30)$ & $-0.32(0.30)$ & $-0.30(0.30)$ \\
\hline
\end{tabular}

Adjusted for gender, age, the level of education, body mass index (continuous), and the use of anti-inflammatory drugs

'Default (non-informative) priors of the software MPlus version 8.4

${ }^{\ddagger}$ Refers to a prior with a normal distribution, mean of zero, and variance of five

${ }^{\S}$ Migraine at follow-up as the dependent variable

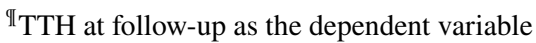

Clinical examination adds to the measurement validity of the TMD-related pain variables, hence increasing the reliability of the effect estimates. A follow-up period of 11 years can be considered a strength in certain respect, namely justifying a sufficient effect of the predictors on the conditions with a slow onset, such as severe headaches. The utilization of DAGs for the appropriate selection of covariates/ confounders to be entered in the regression models can also be considered a strength of the current study. DAGs aid in determining the unbiased associations between the predictor and the outcome variables. Another strength of the study is depicted through application of the Bayesian sensitivity analyses, which demonstrated the stability of the parameter estimates between the variables. This stability measure denotes a sufficient reliability of the parameter estimates of the models. Furthermore, the posterior distribution of the effect estimates places higher credibility on parameter values that are more consistent with the data, i.e., more stable [21].

The current study naturally has also limitations. An obvious shortcoming is the lack of psychosocial aspect (the RDC/TMD Axis II criteria) in the TMD examination. However, this was taken into consideration by adding the anti-inflammatory drug usage as a covariate, since a correlation has been reported between the anti-inflammatory drug usage and the psychological status of migraine [22] and TTH [23] patients. In addition, although the long follow-up period has merits, the lack of measurement time points during the follow-up period must be considered a limitation. The remitting and recurring nature of both TMD-related pain and severe headaches may have caused fluctuations possibly biasing the parameter estimates, a problem not so obvious with more stable exposures and outcomes. The outcome variables assessed by self-report, although being based on a physician diagnosis of severe headaches, can also be considered a limitation. For instance, due to the lengthy follow-up period, recall bias may arise of participants not remembering their diagnoses correctly in the case of rare, intermittent headaches. Moreover, the dichotomous nature of the outcome variables, as well as the low proportion of chronic migraineurs in the current study sample, may also be regarded as limitations of the current study. One possible source of bias may also arise from the overlap between mTMD and TTH; however, the effect of this should minimal, since all the participants with TTH at baseline were excluded and only $7 \%$ of the TTH sufferers at follow-up had both mTMD and TTH. Lastly, although thorough statistical inferential techniques for robust transparency of the results were utilized, these effect estimates could not be interpreted beyond the causal association paradigm.

The current study is, according to the authors' knowledge, the first prospective study reporting the effect estimates between different TMD-related pain variables and the presence of migraine and TTH through Bayesian methodology. Bayesian methodology, which compared to its Frequentist counterpart, provides better estimations in situations of non-random or nested study samples [24]. Utilization of the Bayesian sensitivity analyses also allows for a transparent, yet robust, assessment of the validity and reliability of the effect estimates.

The Bayesian methodology employed in the present study offers several advantages over the traditional Frequentist methodology. This analytical approach produces a range 
of values (Bayesian 95\% CI) as posterior distributions that reflect the uncertainty inherent for the multiple unknown parameters, rather than a fixed value for parameter estimates as in Frequentist approaches. Also, the Bayesian statistical analyses allow for the sub-analyses without the need for the classic statistical adjustments for multiple comparisons. The Bayesian parameter estimates may remain less biased and hence more appropriate and stable with moderate and even smaller sample sizes [25]. Additionally, the interpretation employed to define 95\% confidence intervals in the Frequentist statistics is, in fact, a Bayesian definition for the $95 \%$ CI estimation. The interpretation of the Bayesian 95\% CI states a $95 \%$ probability that the population parameter lies within its defined range [21]. Bayesian methodology should, therefore, be utilized on a more regular basis for better and unbiased effect estimates, especially with smaller or partially non-random nested samples, such as in the current study. As fully informative priors could not be utilized in the current study, Bayesian sensitivity analyses aided in placing meaningful boundaries on the conclusions. These boundaries are needed when there is uncertainty in the choice of priors [26].

The current study found no association between TMDrelated pain (mTMD and jTMD) at baseline and the presence of migraine at follow-up. Only one study [27], to our knowledge, has reported a prospective association between TMD-related pain and migraine. However, that study differed from this study in several aspects such as having a distinctly smaller and selected sample of participants in a tertiary health care facility $v s$. population-based sample in this study. Another difference was the clearly larger proportion of chronic migraineurs in the study population of that study compared to the current study population $(52.3 \% \mathrm{vs} .1 .8 \%$, respectively). Increased pain sensitivity and lowered pressure pain thresholds have been reported along with higher frequency of migraine headaches [28]. These differences may explain the contrasting association patterns between the current study and the study by Stuginski-Barbosa et al. (2010).

The prospective association of mTMD at baseline with the presence of TTH at follow-up found in the current study is in line with previous epidemiological, clinical, and physiological evidence. Previous epidemiological studies have shown an association between TMD-related pain and TTH [29]. Clinically, TMD-related pain and TTH share a combination of distinct signs and symptoms in the head and face region, particularly evident as regards to mTMD and TTH. These common clinical features include palpation tenderness of the masticatory muscles in the case of MTMD and the pericranial muscles in the case of TTH during the active phases of both conditions [30]. Other clinical intersections between mTMD and TTH include subjects' age regarding the peak prevalence [31], the intensity of pain, pharmacotherapy [32], and even the non-pharmacological treatment
[33]. In spite of some clinical similarities and overlap, both mTMD and TTH are distinct disease entities. Although the mix of similarities may necessitate a close interdisciplinary co-operation between specialties (dentistry $v s$. neurology), vigilance should also be exercised regarding the distinction between these two disease entities during their treatment.

In terms of autonomic dysfunction, both mTMD [34] and TTH [35] utilize the trigeminal system to relay nociceptive afferents to their respective higher brain centers. Features shared by MTMD and TTH also include the lower pressure pain threshold and referred pain during their active phases $[34,35]$. These features reflect the presence of peripheral and central sensitization in their respective trigeminal nociceptive pathways. A recent study also reported a high prevalence of active myofascial trigger points in TTH patients [36]. This finding may support the hypothesis that peripheral muscular mechanisms are involved in the pathophysiology of TTH.

Current research reports a significant role of central sensitization in the pathogenesis of chronic TTH [37]. Continuous painful episodes involving pericranial muscles (such as the temporal muscle) may hypersensitize the central nervous system activating the higher brain centers leading to chronic transformation of TTH [38]. This role of mTMD in the chronicity of TTH should always be considered by the dentists and neurologists while treating MTMD and TTH, respectively.

The finding of no association between jTMD and TTH in the current study is in accordance with the findings of a cross-sectional study by Gonçalves et al. (2011) [39]. This may be due to mTMD being more of a generalized pain condition with central sensitization as an important component of its pathogenesis [40], like with the pathogenesis of TTH [29]. Unlike mTMD, jTMD has been considered a localized pathology [41], thus perhaps having a minor effect on the pathogenesis of TTH.

\section{Conclusions}

The current study reported a prospective association of mTMD, but not jTMD, with the presence of TTH. However, TMD-related pain was not found to be associated with the presence of migraine. These results were found reliable after the application of Bayesian sensitivity analyses. An interpretation of these results may be the existence of diverse mechanisms playing a role in the associations between different TMD-related painful conditions and the presence of severe headaches.

Acknowledgements The present study is part of the Health 2000 and 2011 Surveys, organized by the National Institute for Health and Welfare in Finland, and partly supported by the Finnish Dental Society, 
Apollonia, and the Finnish Dental Association. The corresponding author has an early-stage researcher position at the University of Eastern Finland. Furthermore, the corresponding author was a recipient of a grant by the Minerva Foundation, Helsinki, Finland, for attending introductory courses on the Mplus software and the Bayesian statistics.

Author contribution Javed Ashraf: Contributed to conception, design, data acquisition, and interpretation; performed all statistical analyses; and drafted the manuscript.

Matti Närhi: Contributed to conception, design, and data interpretation, and critically revised the manuscript.

Anna Liisa Suominen: Contributed to conception, design, data acquisition, and interpretation, and critically revised the manuscript.

Tuomas Saxlin: Contributed to conception, design, and data interpretation, and critically revised the manuscript.

All authors gave their final approval and agree to be accountable for all aspects of the work.

Funding Open access funding provided by University of Eastern Finland (UEF) including Kuopio University Hospital.

\section{Declarations}

Conflict of interest The authors declare no competing interests.

Open Access This article is licensed under a Creative Commons Attribution 4.0 International License, which permits use, sharing, adaptation, distribution and reproduction in any medium or format, as long as you give appropriate credit to the original author(s) and the source, provide a link to the Creative Commons licence, and indicate if changes were made. The images or other third party material in this article are included in the article's Creative Commons licence, unless indicated otherwise in a credit line to the material. If material is not included in the article's Creative Commons licence and your intended use is not permitted by statutory regulation or exceeds the permitted use, you will need to obtain permission directly from the copyright holder. To view a copy of this licence, visit http://creativecommons.org/licenses/by/4.0/.

\section{References}

1. Ryan J, Akhter R, Hassan N, Hilton G, Wickham J, Ibaragi S (2019) Epidemiology of temporomandibular disorder in the general population : a systematic review. Adv Dent Oral Health 10:1-13. https://doi.org/10.19080/ADOH.2019.10.555787

2. Al-Jundi MA, John MT, Setz JM, Szentpétery A, Kuss O (2008) Meta-analysis of treatment need for temporomandibular disorders in adult nonpatients. J Orofac Pain 22:97-107

3. Qvintus V, Sipilä K, Le Bell Y, Suominen AL (2020) Prevalence of clinical signs and pain symptoms of temporomandibular disorders and associated factors in adult Finns. Acta Odontol Scand 78:515-521

4. GBD (2019) Diseases and injuries collaborators (2020) global burden of 369 diseases and injuries in 204 countries and territories, 1990-2019: a systematic analysis for the global burden of disease study 2019. Lancet 396:1204-1222

5. James SL, Abate D, Abate KH, Abay SM, Abbafati C et al (2018) Global, regional, and national incidence, prevalence, and years lived with disability for 354 diseases and injuries for 195 countries and territories, 1990-2017: a systematic analysis for the Global Burden of Disease Study 2017. Lancet 392(10159):1789-1858
6. Korolainen MA, Kurki S, Lassenius MI et al (2019) Burden of migraine in Finland: health care resource use, sick-leaves and comorbidities in occupational health care. J Headache Pain 20:13. https://doi.org/10.1186/s10194-019-0964-5

7. Raggi A, Leonardi M (2015) Burden and cost of neurological diseases: a European North-South comparison. Acta Neurol Scand 132:16-22. https://doi.org/10.1111/ane.12339

8. Plesh O, Adams S, Gansky S, Plesh O (2011) Temporomandibular joint and muscle disorder (TMJMD) -type pain and co-morbid pains in a national US sample. J Orofac Pain 25:190-198

9. Rantala MAI, Ahlberg J, Suvinen TI, Nissinen M, Lindholm H, Savolainen A, Könönen M (2003) Temporomandibular joint related painless symptoms, orofacial pain, neck pain, headache, and psychosocial factors among non-patients. Acta Odontol Scand 61:217-222

10. List T, Jensen RH (2017) Temporomandibular disorders: old ideas and new concepts. Cephalalgia 37:692-704

11 Ashraf J, Zaproudina N, Suominen A, Sipilä K, Närhi M, Saxlin T (2019) Association between temporomandibular disorders pain and migraine: results of the Health 2000 Survey. J Oral Facial Pain Headache 33(4):399-407. https://doi.org/10.11607/ofph.2213

12. Buchinsky FJ, Chadha NK (2017) To P or not to P: backing Bayesian statistics. Otolaryngol Head Neck Surg 157(6):915-918. https://doi.org/10.1177/0194599817739260

13. Depaoli S, van de Schoot R (2014) Bayesian analyses: where to start and what to report. Eur Heal Psychol 16:75-84

14. Aromaa A, Koskinen S (2004) Health and functional capacity in Finland. Baseline results of the Health 2000 Health Examination Survey. Publications of the National Public Health Institute B12/2004. Helsinki

15. Lundqvist A, Mäki-Opas T (2016) Health 2011 Survey - methods. Juvenes Print - Finnish University Print Ltd. Tampere, Finland

16. Dworkin SF, LeResche L (1992) Research diagnostic criteria for temporomandibular disorders: review, criteria, examinations and specifications, critique. J Craniomandib Disord 6:301-355

17. Suominen-Taipale L, Nordblad A, Vehkalahti M, Arpo Aromaa E (2008) Oral health in the Finnish adult population Health 2000 Survey. Publications of the National Public Health Institute, Report No: B $25 / 2008$. Hakapaino Oy, Helsinki

18. Heistaro S (2008) Methodology report Health 2000 Survey. Publications of the National Public Health Institute, B 26 / 2008. Hakapaino Oy, Helsinki

19. VanderWeele TJ (2019) Principles of confounder selection. Eur J Epidemiol 34:211-219

20. Muthen BO, Muthen LK, Asparouhov T (2016) Regression and mediation analysis using MPlus. Muthen Muthen, Los Angeles

21. Kruschke JK, Liddell TM (2018) Bayesian data analysis for newcomers. Psychon Bull Rev 25:155-177

22. Minen MT, Begasse De Dhaem O, Kroon Van Diest A et al (2016) Migraine and its psychiatric comorbidities. J Neurol Neurosurg Psychiatry 87:741-749

23. Jensen RH (2018) Tension-type headache - the normal and most prevalent headache. Headache 58:339-345

24. Greenland S (2006) Bayesian perspectives for epidemiological research: I. Foundations and basic methods. Int J Epidemiol 35:765-775

25. Shin JJ, Zurakowski D (2017) Null hypotheses, interval estimation, and Bayesian analysis. Otolaryngol - Head Neck Surg 157:919-920

26. Kruschke JK, Liddell TM (2018) The Bayesian new statistics: hypothesis testing, estimation, meta-analysis, and power analysis from a Bayesian perspective. Psychon Bull Rev 25:178-206

27. Stuginski-Barbosa J, MacEdo HR, Eduardo Bigal M, Speciali JG (2010) Signs of temporomandibular disorders in migraine patients: a prospective, controlled study. Clin J Pain 26:418-421 
28. Buchgreitz L, Lyngberg AC, Bendtsen L, Jensen R (2006) Frequency of headache is related to sensitization: a population study. Pain 123:19-27

29. Ciancaglini R, Radaelli G (2001) The relationship between headache and symptoms of temporomandibular disorder in the general population. J Dent 29:93-98

30. Bendtsen L, Ashina S, Moore A, Steiner TJ (2016) Muscles and their role in episodic tension-type headache: implications for treatment. Eur J Pain 20:166-175

31. Costa Y-M, Porporatti A-L, Calderon P-S, Conti P-C-R, Bonjardim L-R (2016) Can palpation-induced muscle pain pattern contribute to the differential diagnosis among temporomandibular disorders, primary headaches phenotypes and possible bruxism? Med oral, Patol oral y cirugía bucal 21:e59-65

32. Neblett R, Cohen H, Choi Y, Hartzell MM, Williams M, Mayer TG, Gatchel RJ (2013) The central sensitization inventory (CSI): establishing clinically significant values for identifying central sensitivity syndromes in an outpatient chronic pain sample. J Pain $14: 438-445$

33. Fernández-De-Las-Peñas C, Cuadrado ML (2016) Physical therapy for headaches. Cephalalgia 36:1134-1142

34. Cairns BE (2010) Pathophysiology of TMD pain-basic mechanisms and their implications for pharmacotherapy. J Oral Rehabil 37:391-410

35. Bendtsen L, Jensen R (2006) Tension-type headache: the most common, but also the most neglected, headache disorder. Curr Opin Neurol 19:305-309
36. Do TP, Heldarskard GF, Kolding LT, Hvedstrup J, Schytz HW (2018) Myofascial trigger points in migraine and tension-type headache. J Headache Pain 19:84. https://doi.org/10.1186/ s10194-018-0913-8

37. Yu S, Han X (2014) Update of chronic tension-type headache. Curr Pain Headache Rep 19:469. https://doi.org/10.1007/ s11916-014-0469-5

38. Bendtsen L, Ashina S, Moore A et al (2016) Muscles and their role in episodic tension-type headache: implications for treatment. Eur J Pain 20:166-175. https://doi.org/10.1002/ejp.748

39. Gonçalves DAGG, Camparis CM, Speciali JG, Franco AL, Castanharo SM, Bigal ME (2011) Temporomandibular disorders are differentially associated with headache diagnoses: a controlled study. Clin J Pain 27:611-615

40. Yunus MB (2008) Central sensitivity syndromes: a new paradigm and group nosology for fibromyalgia and overlapping conditions, and the related issue of disease versus illness. Semin Arthritis Rheum 37:339-352

41. Furquim BD, Flamengui LMSP, Conti PCR (2015) TMD and chronic pain: a current view. Dental Press J Orthod 20:127-133

Publisher's note Springer Nature remains neutral with regard to jurisdictional claims in published maps and institutional affiliations. 